
2

\title{
The Earth and Moon impact flux increased at the end of the Paleozoic
}

\author{
Authors: Sara Mazrouei ${ }^{1 *}$, Rebecca R. Ghent ${ }^{1,2}$, William F. Bottke ${ }^{3}$, Alex H. Parker ${ }^{3}$,
} Thomas M. Gernon ${ }^{4}$

\section{Affiliations:}

${ }^{1}$ Department of Earth Sciences, University of Toronto, Toronto, ON, Canada.

${ }^{2}$ Planetary Science Institute, Tucson, AZ, USA.

${ }^{3}$ Southwest Research Institute, Boulder, CO, USA.

${ }^{4}$ Ocean and Earth Science, University of Southampton, Southampton, United Kingdom.

*Correspondence to: sara.mazrouei.seidani@mail.utoronto.ca

\begin{abstract}
The terrestrial impact crater record is commonly assumed to be biased, with erosion thought to eliminate older craters, even on stable terrains. Given that the same projectile population strike the Earth and Moon, terrestrial selection effects can be quantified using a method that can date diameter $\geq 10 \mathrm{~km}$ lunar craters younger than 1 Gyr. We find the impact rate increased by a factor of 2.6 about 290 million years ago. The terrestrial crater record shows similar results, suggesting the deficit of large terrestrial craters between 300 and 650 million years ago stems from a lower impact flux, not preservation bias. The almost complete absence of terrestrial craters older than 650 million years may indicate a massive global-scale erosion event near that time.
\end{abstract}

One Sentence Summary: An analysis of lunar and terrestrial craters reveals that a substantial increase in larger impacts began about 290 million years ago.

Main Text: The abundance of terrestrial craters with diameter $\geq 20 \mathrm{~km}$ decreases substantially as we go back in time. A common assumption is that this loss is driven by erosive and tectonic processes operating over hundreds of millions of years (Myr). Unfortunately, it is challenging to quantitatively test this hypothesis with existing terrestrial data. An alternative is to estimate terrestrial crater loss rates by comparing Earth's crater record to the Moon's. The Earth and Moon have been struck by the same impactor population over time, but large lunar craters have experienced limited degradation over billions of years. The main obstacle to performing this test has been finding an accurate way to date large lunar craters.

An analysis of the thermophysical characteristics of lunar impact ejecta as measured by the Diviner thermal radiometer on NASA's Lunar Reconnaissance Orbiter $(1,2)$ has now provided the means to estimate the ages of diameter $>10 \mathrm{~km}$ craters younger than 1 Gyr. The formation of large lunar craters excavates numerous $\geq 1 \mathrm{~m}$ ejecta fragments onto the Moon's surface. These newly exposed rocks have high thermal inertia and remain warm during the lunar night relative to the surrounding lunar soils (called regolith) with low thermal inertia. Their nighttime temperatures can be calculated from three of Diviner's thermal infrared channels. Rock abundance values, defined as the fractional coverage of a Diviner pixel by exposed meter-scale rocks (Fig. 1), are obtained, 
simultaneously with rock-free lunar regolith temperatures, by exploiting the fact that a mixture of lunar rocks and regolith produces a mixed spectral radiance and therefore different estimates of brightness temperature in each of the three thermal infrared channels (1).

Using these data, an inverse relationship between rock abundance in large crater ejecta and crater age has been demonstrated by calculating ejecta rock abundance values for nine "index" craters with independently determined ages (2). Young craters are found to have high rock abundance in their ejecta, whereas rock abundance decreases with increasing crater age, eventually becoming indistinguishable from the background for craters older than $\sim 1$ Gyr. The breakdown of lunar rocks has most likely occurred at a steady rate over the last billion years via the constant influx of tiny impactors and the thermal effects of lunar day-night cycling (3). We derive a crater age-rock abundance regression function shown in Fig. 1 and Fig. S1 (3).

We identified 111 rocky craters on the Moon with diameter $\geq 10 \mathrm{~km}$ between $80^{\circ}$ $\mathrm{N}$ and $80^{\circ} \mathrm{S}$, with ejecta blankets that have rock abundance values high enough to distinguish them from the background regolith (Fig. 2a, Table S1). We use the $95^{\text {th }}$ percentile rock abundance values $\left(\mathrm{RA}_{95 / 5}\right)$, which are those that separate the upper $5 \%$ from the lower $95 \%$ of RA values for a given crater's ejecta. We chose $10 \mathrm{~km}$ as a minimum size for this analysis because those craters have penetrated the surface regolith deeply enough to excavate large blocks from the underlying bedrock. This approach minimizes the influence of variations in original ejecta block population due to spatial variations in surface soil thickness $(4,5)$.

Using Fig. 1, we calculated ages for these craters and found that they were not formed uniformly with time (Fig. 2b). This implies the small and large body impact fluxes striking the Moon are probably decoupled from one another at a modest level, with small impactors more likely to maintain a steady impact flux than large impactors (3) (see Fig. S2). Our analysis also showed no statistical evidence for a leading versus trailing hemisphere asymmetry in the calculated ages of these large craters, nor for a latitudinal dependence in rocky crater abundance, though our relatively small sample size might make such a trend difficult to detect. We also identified no correlation between crater sizes and crater ages, meaning differently sized craters are randomly distributed in time.

To quantify the change in flux exhibited by these lunar craters, we adopted a piecewise-constant rate model in which a uniform cratering rate at early times changes instantaneously to a different rate at later times. Sampling from among all possible values of the crater age-rock abundance regression parameters, using conservative estimates on the lunar index crater ages (Fig. 1) (3), we found that this model shows statistical evidence for a break at some time between 220 and $770 \mathrm{Myr}$ (95\% credible intervals), with the peak of the marginalized probability density function (PDF) at a break age of $400 \mathrm{Myr}$ (Fig. S1). The ratio of the crater rate after the break age to the pre-break rate is 2.1 , with $95 \%$ credible interval values of 1.4-20.6.

Supporting evidence for a factor of 2 to 3 increase in the lunar impact flux since $\sim 400$ Myr may come from the ages and abundances of lunar impact spherules. Created by energetic cratering events, these glassy melt droplets have been identified in the regolith samples returned from the Apollo landing sites. Their age distribution is a potential proxy for the impact flux of larger bodies and it suggests the impact flux increased by a factor of $3.7 \pm 1.2$ over the last $400 \mathrm{Myr}(6,7)$, in broad agreement with our results. However, 
the abundance of young impact spherules found in Apollo lunar regolith samples could be a bias (e.g., 7). Lunar craters formed over the last 300-400 Myr may have also degraded faster via diffusion processes than those that formed between 700-3100 Myr (8). This observation may be explained if large impacts enhance diffusive processes via, e.g., seismic shaking, and the large body impact flux has increased over recent times.

Rayed lunar craters have previously been used to compute impact flux rates, with the assumption often made that they formed in the last 1 Gyr. We find 11 farside rocky craters with $D \geq 20 \mathrm{~km}$ formed in the last $1 \mathrm{Gyr}$, compared to 28 to 32 farside rayed craters assumed to be this age $(9,10)$. This discrepancy suggests rayed craters may have a much wider spread of ages than commonly thought (see Supplementary Text).

These results for the Moon provide insights into Earth's crater record. Interpretation of the terrestrial record is problematic because: (i) an unknown number of older craters have been erased at unknown times by erosion or tectonics, (ii) stable continental surfaces capable of recording ancient impacts have potentially been buried and exhumed multiple times since they formed, (iii) it is difficult to precisely quantify which terrains have been adequately searched for craters, and (iv) not all craters are exposed at the surface, but instead have to be identified through geophysical anomalies and explored through drilling (e.g., 11).

Lunar craters have experienced comparatively little erosion over the last $1 \mathrm{Gyr}$, and the proximity of the Earth to the Moon implies that both have been struck by the same population of impactors. A comparison of records on both bodies therefore provides an opportunity to quantify terrestrial selection effects.

Contrary to our expectation, we find that the size-frequency distributions of the lunar and terrestrial craters for diameter $\geq 20 \mathrm{~km}$, normalized by the total number of craters, are highly similar (Fig. S3a). We find no evidence for size bias in retention of terrestrial craters; in an average sense, for a given region, it appears the Earth either keeps all or loses all of its $D \geq 20$ craters at the same rate, independent of size.

We compared the ages of the 38 known terrestrial diameter $D \geq 20 \mathrm{~km}$ craters (Table S2) with the computed age distribution for lunar craters with $\geq 10 \mathrm{~km}$ and $\geq 20 \mathrm{~km}$ (Table S1) (Fig. 3). Using the same statistical method for the terrestrial craters as for the lunar craters, we find that the terrestrial craters also have a break age and present-day to past crater rate ratio close to lunar values (Fig. S1). As there is evidence for a nonuniform terrestrial cratering rate similar to the lunar cratering rate, and considering that the Earth and Moon share a similar bombardment history, we combined both records. The inclusion of terrestrial craters provides an absolute age chronology supplement to the nine index craters we have for the Moon.

The model adopted to fit these data includes a single break between two uniform rates, but we do not rule out other simple models (e.g., cratering rate linearly increasing in time) or more complex models (e.g., multiple breaks). Rather, we use the single-break piecewise model as a simple and physically plausible hypothesis to demonstrate that the lunar and terrestrial cratering rates have not been constant over the past billion years.

Our joint lunar and terrestrial analysis yields a ratio of the crater rate after the break age to the pre-break rate of 2.6 , with a $95 \%$ credible interval value of $1.7-4.7$. The most probable break age is $290 \mathrm{Myr}$. The impact rate change is reflected in the sizefrequency distribution curves, with craters younger than 290 Myr substantially higher in frequency at all diameters than those older than 290 Myr (Fig. 2b). The deficit of large 
terrestrial craters between 290-650 Myr can therefore be interpreted to reflect a lower impact flux relative to the present day and not a bias (Supplementary Text).

The erosion history of Earth's continents can also be constrained using uraniumlead $(\mathrm{U}-\mathrm{Pb})$ thermochronology, or temperature-sensitive radiometric dating. burial rates of up to $2.5 \mathrm{~m} \mathrm{Myr}^{-1}$ (12), which equates to a maximum of $1.6 \mathrm{~km}$ vertical erosion (or deposition) over the last $650 \mathrm{Myr}$. This would likely be insufficient to eradicate $\geq 20 \mathrm{~km}$ craters, given that crater depths are approximately equal to $\sim 10 \%$ of their original diameter (13).

Support for limited erosion on cratered terrains can also be found in the record of kimberlite pipes. Kimberlites are formed during explosive volcanism from deep mantle sources, generating carrot-shaped pipes 1-2 km deep (14, 15) (Fig. 4), and commonly preserve volcanic features (e.g. volcanic craters, pipes) that are depth-diagnostic (16). Impact craters and kimberlites are frequently found in common regions on stable continental surfaces (Fig. 4a), so kimberlites are a proxy indicating the depth of erosion for surfaces of different ages. Deep erosion of stable continental surfaces $(>2 \mathrm{~km})$ should have removed most kimberlite pipes, leaving behind deep-seated intrusive rocks, but kimberlite pipes are relatively common throughout the Phanerozoic Eon (541 million years ago to the present). Their spatio-temporal distribution (Fig. 4b) suggests only modest erosion $(<1 \mathrm{~km})$ on most cratons since $650 \mathrm{Myr}$, favoring the survival of $\geq 20 \mathrm{~km}$ impact craters (3).

There is a sharp cut-off in the number of terrestrial craters at $\sim 650 \mathrm{Myr}$ (Figs. 34). Given erosion rates on stable continental terrains after $650 \mathrm{Myr}$, similar conditions further back in time would have allowed most craters of Precambrian age (i.e., older than $541 \mathrm{Myr}$ ) to survive. Instead, the paucity of Precambrian craters is coincident with major episodes of globally extensive 'Snowball Earth' glaciation (17) (Fig. 4b). Pervasive subglacial erosion at $\sim 650-720 \mathrm{Myr}$ is thought to have removed kilometers of material from the continents $(18,19)$, enough to erase most existing kimberlite pipes and impact craters (Fig. S5a). The exceptions are the $>130 \mathrm{~km}$ impact craters Sudbury (1850 Myr) and Vredefort (2023 Myr). Both craters were deep enough to survive, but each shows indications of multiple kilometers of erosion (20).

The change in the lunar and terrestrial impact flux may be due to the breakup of one or more large asteroids in the inner and/or central main asteroid belt (e.g., 21). Those located near dynamical resonances may produce long-lived surges in the impact flux as the fragments are slowly driven to escape routes by non-gravitational forces. Asteroid evolution models suggest the contribution of kilometer-sized impactors from a large parent body disruption would have reached their new level within a few tens of Myr of the breakup event(s), with the wave of bodies perhaps receding after hundreds of Myr $(21,22)$.

\section{References and Notes:}

1. J. L. Bandfield, et al., Lunar surface rock abundance and regolith fines temperatures derived from LRO diviner radiometer data. Journal of Geophysical Research: Planets. 116, E00H02 (2011).

2. R. R. Ghent, et al., Constraints on the recent rate of lunar ejecta breakdown and implications for crater ages. Geology. 42, 1059-1062 (2014). 
3. Materials and methods are available as supplementary materials.

4. G. H. Heiken, D. T. Vaniman, B. M. French, Lunar sourcebook - A user's guide to the Moon (Cambridge University Press, England, 1991).

5. R. J. Pike, Depth/diameter relations of fresh lunar craters - Revision from spacecraft data. Geophysical Research Letters. 1, 291-294 (1974).

6. T. S. Culler, A. Timothy, A. M. Richard, P. R. Renne, Lunar impact history from ${ }^{40} \mathrm{Ar} /{ }^{39} \mathrm{Ar}$ dating of glass spherules. Science. 287, 1785-1788 (2000).

7. N. Zellner, J. Delano, ${ }^{40} \mathrm{Ar} /{ }^{39} \mathrm{Ar}$ ages of lunar impact glasses: Relationships among $\mathrm{Ar}$ diffusivity, chemical composition, shape, and size. Geochimica et Cosmochimica Acta 161, 203 - 218 (2015).

8. C. I. Fassett, B. J. Thomson, Crater degradation on the lunar maria: Topographic diffusion and the rate of erosion on the moon. Journal of Geophysical Research: Planets. 119, 2255-2271 (2014).

9. A. S. McEwen, J. M. Moore, E. M. Shoemaker, The Phanerozoic impact cratering rate: Evidence from the farside of the Moon. Journal of Geophysical Research: Planets. 102, 9231-9242 (1997).

10. J. A. Grier, A. S. McEwen, P. G. Lucey, M. Milazzo, R. G. Strom, Optical maturity of ejecta from large rayed lunar craters. Journal of Geophysical Research: Planets. 106, 32847-32862 (2001).

11. R. A. F. Greive, E. M. Shoemaker, "The record of past impacts on Earth", In Hazards Due to Comets and Asteroids (Univ. Arizona Press), pp 417-462 (1994).

12 . T. J. Blackburn, S. A. Bowring, J. T. Perron, K. H. Mahan, F. O. Dudas, K. R. Barnhart, An exhumation history of continents over billion-year time scales. Science. 335(6064), 73-76 (2012).

13. E. I. Smith, Determination of origin of small lunar and terrestrial craters by depth diameter ratio. Journal of Geophysical Research. 76(23), 5683-5689 (1971).

14. R. S. J. Sparks, L. Baker, R. J. Brown, M. Field, J. Schumacher, G. Stripp, A. Walters, Dynamical constraints on kimberlite volcanism. Journal of Volcanology and Geothermal Research. 155(1), 18-48 (2006).

15. L. Wilson, J. W. Head III, An integrated model of kimberlite ascent and eruption. Nature. 447, 53-57 (2007).

16. R. J. Brown, G. A. Valentine, Physical characteristics of kimberlite and basaltic intraplate volcanism and implications of a biased kimberlite record. GSA Bulletin $\mathbf{1 2 5}$ (7-8), 1224-1238 (2013).

17. Hoffman, P. F., Kaufman, A. J., Halverson, G. P. \& Schrag, D. P., A Neoproterozoic Snowball Earth. Science 281, 1342-1346 (1998).

18. C. B. Keller, et al., In Press. Neoproterozoic glacial origin of the Great Unconformity. Proceedings of the National Academy of Sciences (2018).

19. M. S. DeLucia, W.R. Guenthner, S. Marshak, S.N. Thomson, A.K. Ault, Thermochronology links denudation of the Great Unconformity surface to the supercontinent cycle and snowball Earth. Geology 46 (2), 167-170 (2017).

20. R. A. F. Grieve, W. U. Reimold, J. Morgan, U. Riller, M. Pilkington, Observations and interpretations at Vredefort, Sudbury, and Chicxulub: Towards an empirical model of terrestrial impact basin formation. Meteoritics \& Planetary Science. 43(5), 855-882 (2008). 
21. W. F. Bottke, D. Vokrouhlicky, D. Nesvorny, An asteroid breakup $160 \mathrm{Myr}$ ago as the probable source of the K/T impactor. Nature. 449, 48-53 (2007).

22. D. Vokrouhlicky, W. F. Bottke, D. Nesvorny. The Flora family event: Implications for the near-Earth asteroid population and large impactors of terrestrial worlds. Astron $J .153,172$ (2017).

23. M. Zanetti, et al,. Evidence for self-secondary cratering of Copernican-age continuous ejecta deposits on the moon. Icarus. 298, 64 - 77 (2017).

24. "Earth Impact Database". Planetary and Space Science Centre (PASSC), University of New Brunswick. http://www.passc.net/EarthImpactDatabase/. Retrieved 2016-0722.

25. S. Faure, CONSOREM Database (Version 3), Consortium de Recherche en Exploration Minérale CONSOREM, Université du Québec à Montréal, www.consorem.ca (2010). Retrieved 2016-07-22.

26. Geological Survey of Canada, Generalized geological map of the world and linked databases. Technical Report 2915d (1995).

27. United States Geological Survey, Geologic Province Map. https://earthquake.usgs.gov/data/crust/type.html. Retrieved 2018-03-05.

28. J. L. Bandfield, et al., Distal ejecta from lunar impacts: Extensive regions of rocky deposits. Icarus. 283, 282-299 (2017).

29. Paige et al., LRO DLRE LEVEL 5 GDR V1.0, NASA Planetary Data System, LROL-DLRE-5-GDR-V1.0, 2011. https://pds.nasa.gov/dsview/pds/viewDataset.jsp?dsid=LRO-L-DLRE-5-GDR-V1.0. Retrieved 2016-07-22.

30. D. Foreman-Mackey, D. W. Hogg, D. W., Lang, D. \& Goodman, J. emcee: The MCMC hammer. Astronomical Society of The Pacific. 125, 306-312 (2013).

31. J. K. Pritchard, M. T. Seielstad, A. Perez-Lezaun, M. W. Feldman. Population growth of human Y chromosomes: a study of Y chromosome microsatellites. Molecular biology and evolution. 16, 1791-1798 (1999).

32. J. M. Marin, P. Pudlo, C. P. Robert, R. J. Ryder, Approximate Bayesian Computational methods. Statistics and Computing. 1-14 (2012).

33. A. H. Parker, The intrinsic Neptune Trojan orbit distribution: Implications for the primordial disk and planet migration. Icarus. 247, 112-125 (2015).

34. H. Jeffreys, An invariant form for the prior probability in estimation problems. Proceedings of the Royal Society London A, 186, 453-461 (1946).

35. F. W. Scholz, M. A. Stephens, K-Sample Anderson-Darling Tests, Journal of the American Statistical Association, 82, 918-924 (1987).

36. V. C. Sousa, M. Fritz, M. A. Beaumont, L. Chicki, Approximate Bayesian computation without summary statistics: The case of admixture. Genetics. 181, 15071519 (2009).

37. C. Robert, J. M. Cornuet, J. M. Marin, N. Pillai, Lack of confidence in approximate Bayesian computation model choice. Proceedings of the National Academy of Sciences. 108, 15112-15117 (2011).

38. H. Jeffries, Theory of probability (Clarendon Press, Oxford, 1961).

39. G. Neukum, B. A. Ivanov, W. K. Hartmann, Cratering Records in the Inner Solar System in Relation to the Lunar Reference System. Space Science Reviews. 96, 55-86 (2001). 
40. D. Stoffler, et al., Cratering history and lunar chronology. Rev. Mineral. Geochem. 60(1):519-96 (2006).

41. H. Hiesinger, et al., How old are young lunar craters? Journal of Geophysical Research (Planets). 117, E00H10 (2012).

42. S. J. Robbins, New crater calibrations for the lunar crater-age chronology. Earth and Planetary Science Letters. 403, 188 - 198 (2014).

43. J. P. Williams, et al., Dating very young planetary surfaces from crater statistics: A review of issues and challenges. Met. Planet. Sci. 10.1111/maps.12924 (2017).

44. W. F. Bottke, et al., Debiased orbital and absolute magnitude distribution of the nearearth objects. Icarus. 156, 399-433 (2002).

45. W. F. Bottke, D. Vokrouhlicky, D. P. Rubincam, and D. Nesvorny. The Yarkovsky and YORP Effects: Implications for asteroid dynamics. Ann. Rev. Earth Planet. Sci. 34, 157-191 (2006).

46. W. F. Bottke, et al., Linking the collisional history of the main asteroid belt to its dynamical excitation and depletion. Icarus. 179, 63-94 (2005).

47. W. F. Bottke, et al., The Collisional Evolution of the Asteroid Belt. In Asteroids IV (P. Michel, F. DeMeo, and W. F. Bottke, Eds). U. Arizona Press, 701-724 (2015).

48. M. Granvik, et al., Super-catastrophic disruption of asteroids at small perihelion distances. Nature. 530, 303-306 (2016).

49. D. Nesvorný, D. Vokrouhlický, A. Morbidelli, W. F. Bottke. Asteroidal source of L chondrite meteorites. Icarus. 200, 698-701 (2009).

50. J. R. Masiero, A. K. Mainzer, T. Grav, J. M. Bauer, R. Jedicke, Revising the Age for the Baptistina Asteroid Family Using WISE/NEOWISE Data. The Astrophysical Journal. 759, 14 (2012).

51. P. G. Brown, P. Wiegert, D. Clark, E. Tagliaferri, Orbital and physical characteristics of meter-scale impactors from airburst observations. Icarus 266, 96-111. (2016).

52. L. W. Bandermann, S. F. Singer, Calculation of meteoroid impacts on moon and earth. Icarus. 19, 108-113 (1973).

53. D. W. Hughes, A new approach to the calculation of the cratering rate of the Earth over the last $125 \pm 20$ Myr. Monthly Notices of the Royal Astronomical Society. 317, 429-437 (2000).

54. R. A. F. Grieve, E. M. Shoemaker, The Record of Past Impacts on Earth. In T. Gehrels, M. S. Matthews, A. M. Schumann (eds.) Hazards Due to Comets and Asteroids, 417 (1994).

55. E. M. Shoemaker, Impact cratering through geologic time. J. R. Astron. Soc. Can. 92 297-309 (1998).

56. D. E. Wilhelms, with sections by J. F. McCauley, N. J. Trask, The geologic history of the moon. Tech. Rep. (USGS Professional Paper 1348, Denver, 1987).

57. B. R. Hawke, et al., The origin of lunar crater rays. Icarus. 170, 1-16 (2004).

58. P. G. Lucey, D. T. Blewett, G. J. Taylor, B. R. Hawke, Imaging of lunar surface maturity. Journal of Geophysical Research: Planets. 105, 20377-20386 (2000).

59. J. K. Harmon, et al., Mercury: Radar images of the equatorial and midlatitude zones. Icarus. 187, 374-405 (2007).

60. C. D. Neish, et al., A comparison of rayed craters on the Moon and Mercury. Journal of Geophysical Research: Planets. 118, 2247-2261 (2013). 
61. R. H. Mitchell, Kimberlites: Mineralogy, Geochemistry, and Petrology. Plenum Press, (1986).

62. R. M. Flowers, B. Schoene, (U-Th)/He thermochronometry constraints on unroofing of the eastern Kaapvaal craton and significance for uplift of the southern African Plateau. Geology. 38(9), 827-830 (2010).

63. J. Braun, F. Guillocheau, C. Robin, G. Baby, H. Jelsma, Rapid erosion of the Southern African Plateau as it climbs over a mantle superswell. Journal of Geophysical Research: Solid Earth. 119(7), 6093-6112 (2014).

64. C. Johnson and T. J. Bowling. Where have all the craters gone? Earth's bombardment history and the expected terrestrial cratering record. Geology. 42(7), 587-590 (2014).

65. T. H. Torsvik, K. Burke, B. Steinberger, S. J. Webb, L. D. Ashwal, Diamonds sampled by plumes from the core-mantle boundary. Nature. 466(7304), 352-355 (2010).

66. C. Alwmark, W. Bleeker, A. LeCheminant, L. Page, A. Schersten, An Early Ordovician ${ }^{40} \mathrm{Ar}-{ }^{39} \mathrm{Ar}$ age for the ${ }^{\sim} 50 \mathrm{~km}$ Carswell impact structure, Canada. The Geological Society of America. 129 (11-12), 1442-1449 (2017).

67. D. J. Milton, J. F. Sutter, Revised age for the Gosses Bluff impact structure, Northern Territory, Australia, based on ${ }^{40} \mathrm{Ar} /{ }^{39} \mathrm{Ar}$ dating. Meteoritics, 22(3), 281-289 (1987).

68. E. P. Gurov, E. P. Gurova, The Kurakul depression in the Pamirs - a reliable impact structure. Geologicheskii Zhurnal, 6, 53-64 (1993).

69. X. Hu, et al., The timing of India-Asia collision onset - Facts, theories, controversies. Earth-Science Reviews, 160, 264-299 (2016).

70. M. M. Meier, S. Holm-Alwmark, A tale of clusters: no resolvable periodicity in the terrestrial impact cratering record. Monthly Notices of the Royal Astronomical Society, 467(3), 2545-2551 (2017).

71. M. Schmieder, F. Jourdan, E. Tohver, E. A. Cloutis, E.A, ${ }^{40} \mathrm{Ar} /{ }^{39} \mathrm{Ar}$ age of the Lake Saint Martin impact structure (Canada)-Unchaining the Late Triassic terrestrial impact craters. Earth and Planetary Science Letters, 406, 37-48 (2014).

72. F. Jourdan, W. U. Reimold, Age of the Siljan impact structure. Meteoritics and Planetary Science Supplement, 75, abstract no. 5093 (2012).

Acknowledgments: We thank Martin Schmeider and Christian Koeberl for helpful discussions with regard to ages of terrestrial craters, Thea Hincks for her help generating the plots in Figs. 4 and S5, and Jon Husson for providing digital Precambrian bedrock outlines shown in Fig. 4. We thank Brenhin Keller and Ross Mitchell for stimulating discussions concerning Cryogenian erosion. We also thank the anonymous referees for their useful and constructive comments.

Funding: S. Mazrouei and R. R. Ghent's work on this study were funded by a Discovery grant from the National Science and Engineering Research Council of Canada to R. R. Ghent. W. F. Bottke's participation was supported by NASA's SSERVI program "Institute for the Science of Exploration Targets (ISET)" through institute grant number NNA14AB03A. A.H. Parker's participation was supported in part by NASA's SSERVI program "Project for Exploration Science Pathfinder Research for Enhancing Solar System Observations (Project ESPRESSO)" through institute grant number 
361 80ARC0M0008. T. M. Gernon acknowledges funding from the UK Natural Environment

362 Research Council, grant reference NE/R004978/1.

364 Author contributions: R.G. conceived the lunar crater experiments. S.M. collected 365 lunar crater data. Statistical tests were performed by A.P. Expertise on asteroid evolution 366 and impact probabilities was provided by W.F.B. Expertise on kimberlite pipes was 367 provided by T.G. All authors (S.M, R.G., W.F.B, A.P., and T.G.) analyzed the results 368 and wrote the manuscript.

369

Competing interests. The authors have no competing interests.

Data and materials availability: The LRO Diviner data used in this paper can be obtained from (29). The derived lunar crater data are provided in Table S1 and the terrestrial crater data used (updated from (24)) are provided in Table S2. The kimberlite database (updated from (25)) used to generate Fig. 4 and Fig. S5 is provided in Supplementary Data File S1 (aar4058_s1). The Approximate Bayesian Computation rejection ( $\mathrm{ABCr}$ ) code and the counting area simulation code (used to generate Fig. S4) are available at https://github.com/ghentr/Earth-Moon_flux.

\section{SUPPLEMENTARY MATERIALS}

www.sciencemag.org/cgi/content/

Materials and Methods

Supplementary Text

Figs. S1 to S6

Tables S1 to S2

Data File S1 


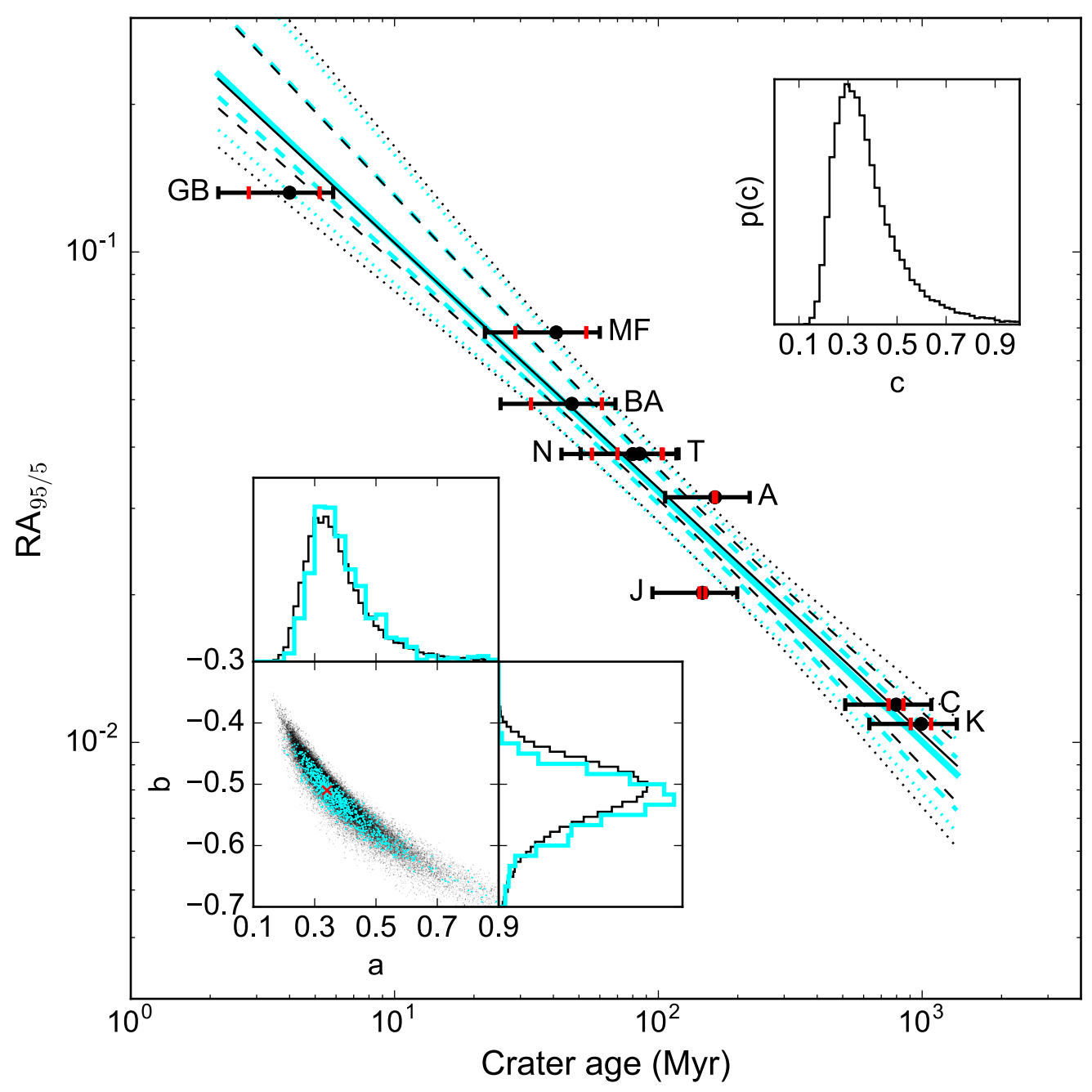

Fig. 1. Regression of lunar crater age versus $95^{\text {th }}$ percentile rock abundance (updated from 1, 2). Data point labels correspond to dated lunar craters (2) listed in Table S1.

392 Rock cover is defined as materials with rock-like thermal inertia and minimum diameters

393 larger than the diurnal thermal skin depth $(\sim 0.5 \mathrm{~m})$. This regression differs from previous

394 analysis (2) due to use of an updated rock abundance dataset and an updated age for

395 Aristarchus crater (23), together with a statistical treatment that marginalizes over

396 unacknowledged uncertainties for the published crater ages (3). Red error bars illustrate uncertainties for each crater, and black error bars show the uncertainties implied by the median value of the uncertainty scaling factor $c$ given its posterior PDF (Equation S2). The best fitting parameters in the relation $\mathrm{RA}_{95 / 5}=a \times(\text { age } / \mathrm{Myr})^{b}$ are $a: 0.33 ; b:-0.50$ (black solid curve); black dashed and dotted curves show the $68 \%$ and $95 \%$ credible intervals. After propagation through the joint terrestrial/ lunar Approximate Bayesian Computation rejection ( $\mathrm{ABCr}$ ) analysis (3), the best fitting parameters are $a: 0.34 ; b$ : intervals. Inset figures show the $2 \mathrm{D}$ distribution of the posterior PDF sample of 
parameters $(a, b)$ before and after $\mathrm{ABCr}$ analysis (black and cyan points, respectively), their marginalized distributions, and $p(c)$, the $1 \mathrm{D}$ marginalized posterior PDF of the uncertainty scaling factor $c$ (Equation S2).
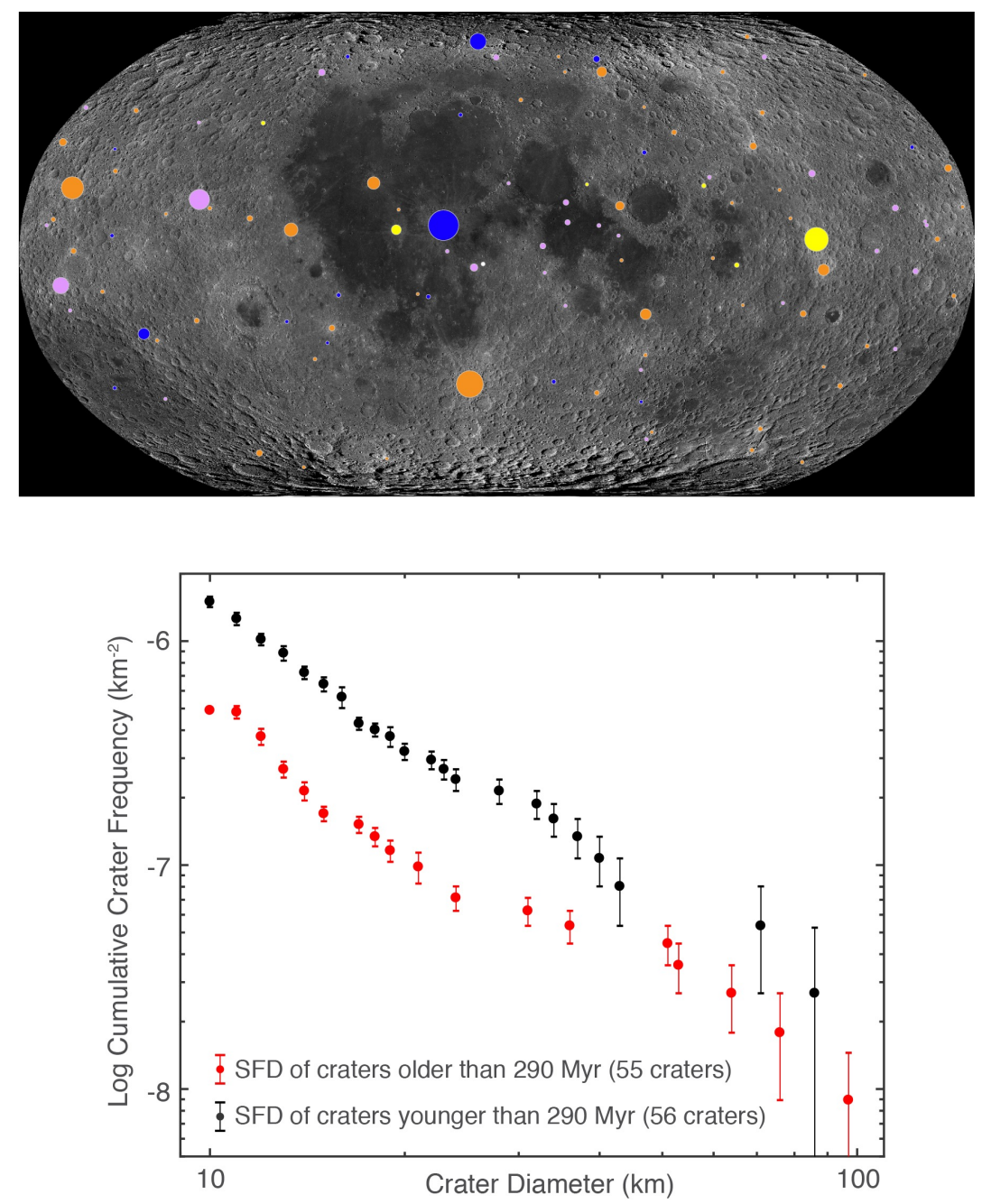

Fig. 2. Geographic and size-frequency distribution of rocky lunar craters. (A) Geographic distribution of 111 rocky (young) craters with diameter $\mathrm{D} \geq 10 \mathrm{~km}$ between $80^{\circ} \mathrm{N}$ and $80^{\circ} \mathrm{S}$ on the Moon (listed in Table S1), scaled by size and color coded according to age. Orange (dark yellow deuteranopia): craters younger than $290 \mathrm{Myr}$; pink (light blue deuteranopia): 290-580 Myr; dark blue: 580-870 Myr; yellow: 870-1160 Myr; white: older than 1160 Myr. Background image is from the 2013 LROC WAC global mosaic (https://www.lroc.asu.edu). (B) Cumulative size-frequency distributions of craters: red: average SFD of craters older than 290 Myr (55 craters; average of cumulative distribution in three age bins: 290-580 Myr; 580-870 Myr; and 870-1160 Myr); black: craters younger than 290 Myr (56 craters), error bars show Poisson noise. The lunar cratering rate has increased by a factor of 2.6 in the last 290 Myr compared to the preceding $\sim 710 \mathrm{Myr}$. 


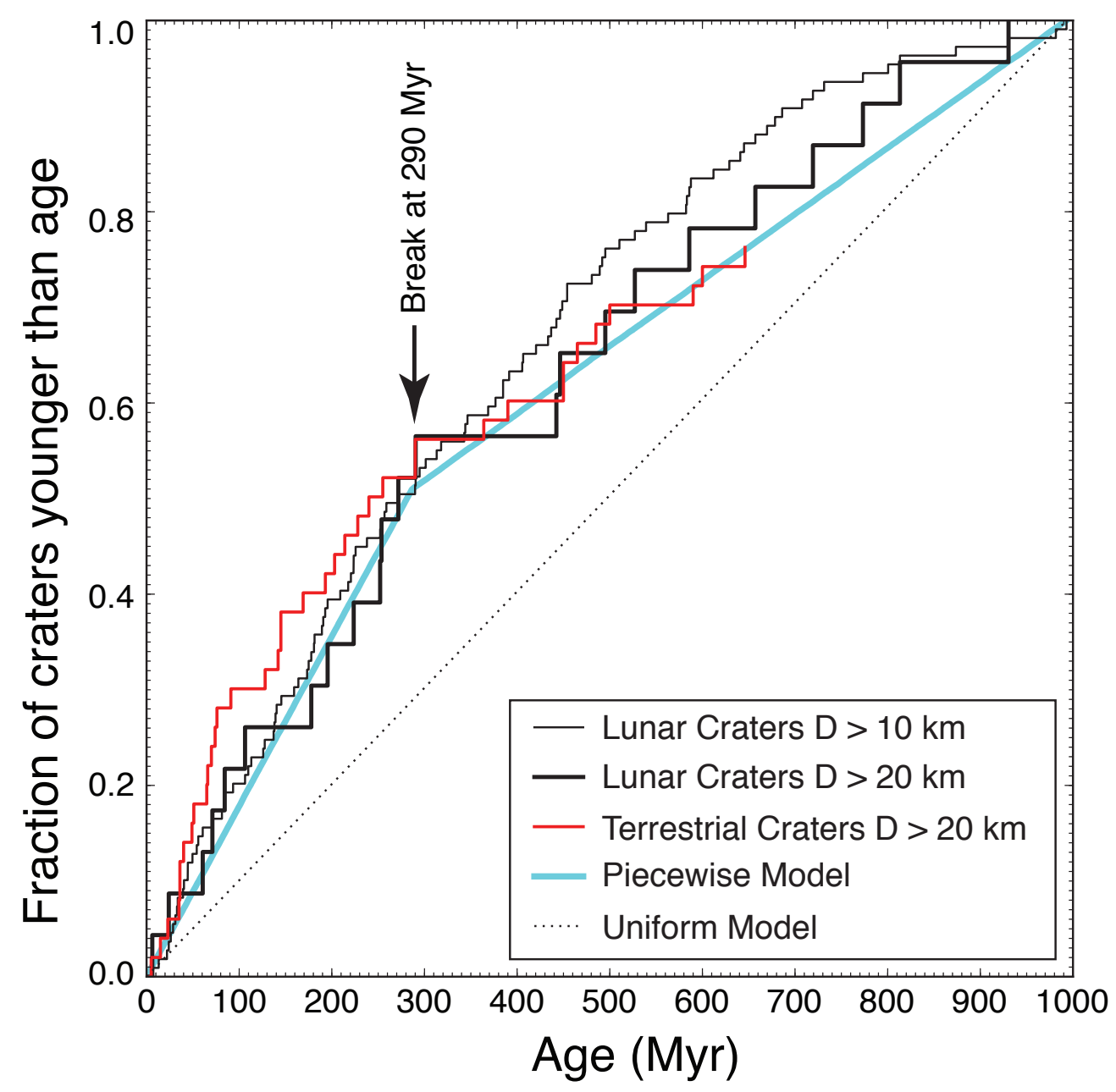
and $20 \mathrm{~km}$ curves are shown by the black line, while $D \geq 20 \mathrm{~km}$ terrestrial craters (Table $\mathrm{S} 2)$ are shown with the red line. All terrestrial craters are younger than $650 \mathrm{Myr}$. The lunar impact flux increases by a factor of 2.6 near $290 \mathrm{Myr}$ (Fig. S1). A simple piecewise model (cyan) demonstrates the break between two rates compared to a simple uniform suggests that the inferred increase in terrestrial impacts is not a preservation bias. 
A
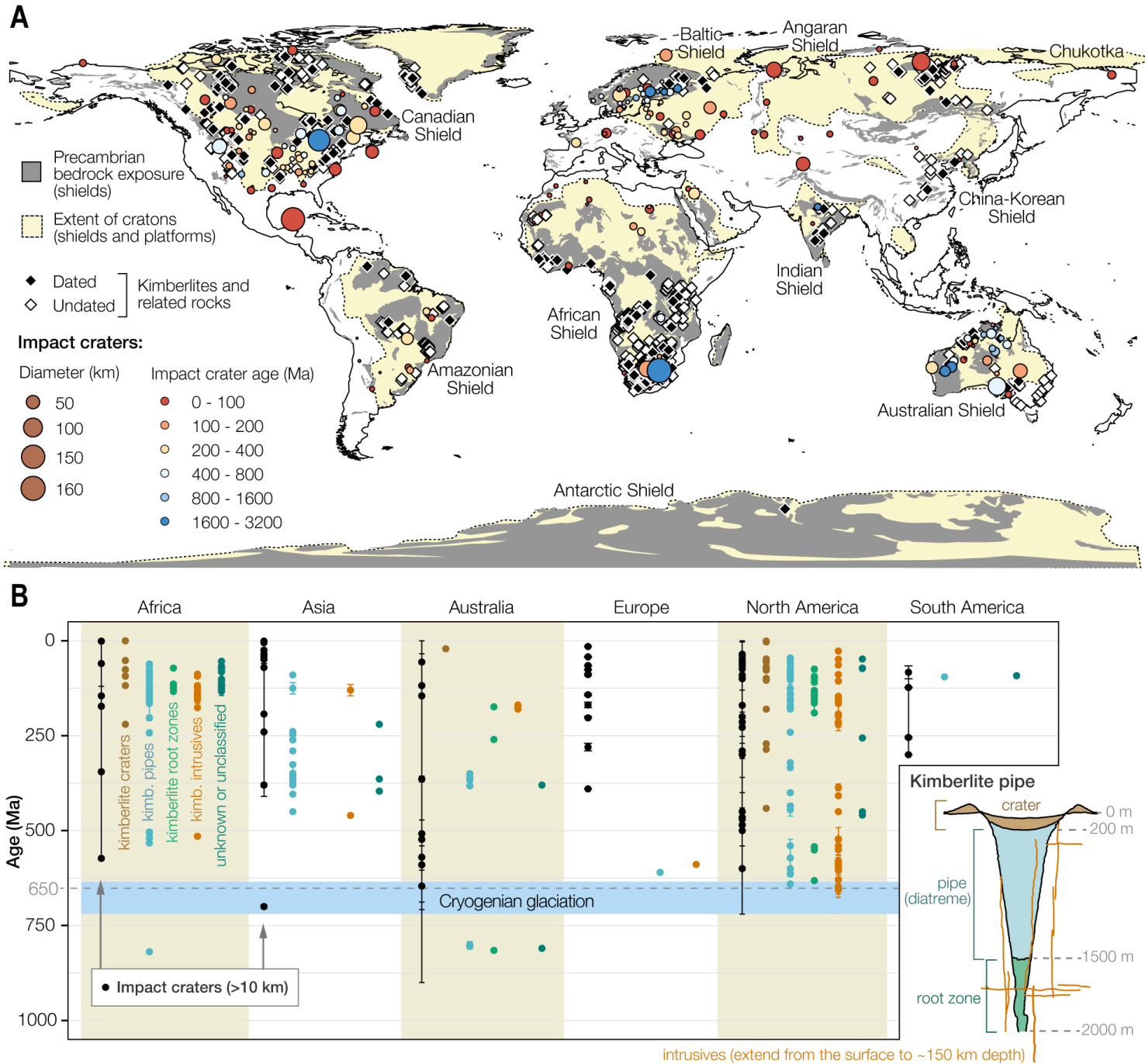

Fig. 4. Positions of terrestrial impact craters and kimberlites in space and time. (A) Locations of all impact craters identified in the Planetary and Space Science Centre (PASSC) Earth Impact Database(24), scaled by size and colored by age. Kimberlite occurrences are also shown; solid symbols denote those with well-defined ages $(n=624)$ and white diamonds show undated kimberlites $(n=3645)(25)$. Gray regions correspond to major exposures of Precambrian basement rocks (26), which together with platform areas shown in beige (27) form the stable cratons, where $84 \%$ of $D \geq 20 \mathrm{~km}$ craters (and $84.6 \%$ of $\mathrm{D} \geq 10 \mathrm{~km}$ craters) occur. (B) Chronology of large impacts ( $>10 \mathrm{~km})$ and welldated kimberlites for each continent, excluding Antarctica. Colored symbols signify depth-diagnostic kimberlite zones (labeled and illustrated in the inset). There is an abrupt cut-off in impact crater and kimberlite pipe frequency at $\sim 650 \mathrm{Myr}$, coincident with Snowball Earth glaciation during the Cryogenian Period, 720 to 635 million years ago (17-19). 
449 Supplementary Materials:

450 Materials and Methods

451 Supplementary Text

452 Figures S1-S6

453 Tables S1-S2

454 Data File S1

455 References (28-72) 